\title{
Design and development virtual exhibition system for museum based on Augmented Reality
}

\author{
Yue $\mathrm{Yu}{ }^{1}$, Shuchang $\mathrm{Xu}^{1+}$ and Qingshu Yuan ${ }^{1}$ and Yongchun Zhang ${ }^{2}$ \\ ${ }^{1}$ College of Information Science and Engineering, Hangzhou Normal University, Hangzhou Zhejiang, China \\ ${ }^{2}$ Zhejiang Provincial Museum, Hangzhou Zhejiang, China
}

\begin{abstract}
The contradiction between taking pictures and exhibits protection and order maintenance has become an urgent problem to be solved by the museum. This paper introduced a virtual exhibition system for museum based on Augmented Reality technology to solve this problem. This paper developed a system based on ARToolKit, repaired the ARToolKit some defects about marker identification and texture mapping and achieved a variety of interactive functions. It conducted a test run in the Zhejiang provincial museum. It had a very good promotion value and application prospects.
\end{abstract}

Keywords: museum; Augmented Reality; mobile phone

\section{Introduction}

Museum is widely favoured by the people as a display and exhibition place of human nature and cultural heritage. Taking pictures in the museum may damage exhibits and affect visit order, therefore a number of museums made it clear that it is not allowed to take pictures in the exhibition hall. The contradiction between taking pictures and exhibits protection and order maintenance has become an urgent problem to be solved by the museum. Using Augmented Reality (AR) technology can enhance user's visual experience, extend the user's vision, interact with the real and virtual integration environment in a more natural way, and complete some tasks better [1].

In the museum, AR technology has been more and more widely used. In the "China Terracotta Warriors" exhibition held at the Asian Art Museum in San Francisco in 2013, the Asian Art Museum launched an AR application software that can display 3D content and video content in the "real environment" on users' phone when users scan the presentation cards in the exhibition hall [2]. In 2015, the Shanghai Natural History Museum and the Shanghai People's Fine Arts Publishing House co-issued an AR calendar. In 2016, the Palace Museum and Qian Tech together published a called "Imperial Life" AR calendar.

With regard to the current AR application in museums, we found that the following two aspects can be further improved. First, the existing system in the marker select method is not so flexible that the marker cannot be personalized customized. Second, the marker of the existing system cannot be self-help but need for users in fixed area or place in the museum to obtain, so the convenience of using app is not good.

The contribution of this paper lies in the following two aspects: First of all, the system proposed in this paper supports flexible marker definition that support for black and white markers and color image markers. Secondly, this article combines the museum's WeChat self-help bookmark printing system, effectively extending the museum's exhibition time and space.

\section{System Framework}

\footnotetext{
+ Corresponding author. Tel.: + 8613732271527.

E-mail address: philous@163.com.
} 
The system framework shown in Fig. 1. After the App identified a marker and showed the 3D model of corresponding cultural relic, users can rotate or scale the virtual cultural relic, view the introduction of the cultural relic and do some other operations.

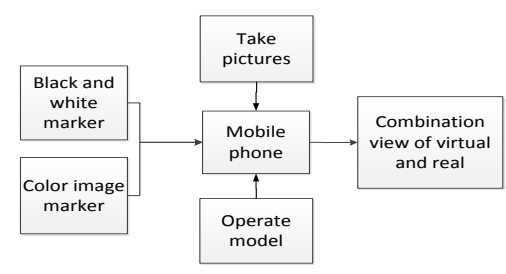

Fig. 1: System framework.

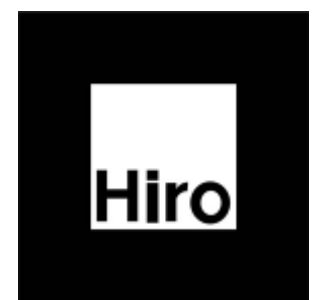

Fig. 2: Black and white marker sample.

\section{Technical details}

The application is based on ARToolKit v5.3.2. In this section, the technical of black and white marker will be introduced in four aspects, and the color image marker scheme will be introduced from three aspects.

\subsection{Technical details of black and white marker}

\subsubsection{Markers creation}

A new marker can be designed and created as shown in Fig. 2. You can change the pattern inside the black box into the pattern you want. After making black and white markers, developers need to use the program "mk_patt" provided by ARToolKit to train markers and generate corresponding pattern recognition data files for this system to detect, identify and track markers.

\subsubsection{Addition of 3D models}

This article will use the ARToolKit example project "ARSimpleNativeCarsProj" to describe and write a new custom application program. Adding a marker-3D model pair by the following function:

models [0].patternID = arwAddMarker("single;Data/hiro.patt;80");

const char *modelOfile = "Data/models/FO.obj";

models [0].obj = glmReadOBJ2 $($ modelOfile, 0,0$)$;

The type of array "model" is ARModel. "Data/hiro.patt" is the path of this marker recognition data files generated in previous section. "Model0file" is the path of the 3D model file.

\subsubsection{Models transform}

ARToolKit can track markers and change models' view transform in real time. The following explains how to get the vector of rotation axis and the model transformation matrix when users want to rotate the model correctly.

The view matrix $T_{M}$ can be obtained from ARToolKit. If the model is rotated counterclockwise around the $\mathrm{x}$-axis, let the $\mathrm{x}$-axis positive vector $u=(1,0,0)$. For the sake of calculation, let $u{ }^{\prime}=(1,0,0,0)$, then $u^{\prime}{ }^{\prime}=T_{M}^{-1} \cdot u^{\prime}$ is the transformed $\mathrm{x}$-axis positive vector. Similarly, let $u=(0,1,0)$, we can get the transformed y-axis positive vector. According to the working principle of ARToolKit [3] [4] and the principle of 3D geometric transformation [5], the rotation equation of the 3D object around z-axis is as follows:

$$
\left[\begin{array}{l}
x^{\prime} \\
y^{\prime} \\
z^{\prime} \\
1
\end{array}\right]=\left[\begin{array}{cccc}
\cos \theta & -\sin \theta & 0 & 0 \\
\sin \theta & \cos \theta & 0 & 0 \\
0 & 0 & 1 & 0 \\
0 & 0 & 0 & 1
\end{array}\right] \cdot\left[\begin{array}{l}
x \\
y \\
z \\
1
\end{array}\right]
$$

Similarly, the rotation equation can be obtained around $\mathrm{x}, \mathrm{y}$ axis. Let the unit vector of the rotation axis be $u=(a, b, c)$, the rotation angle is $\theta$. The transformation matrix of the model is derived according to the following formula:

$$
R(\theta)=T^{-1} \cdot R_{x}^{-1}(\alpha) \cdot R_{y}^{-1}(\beta) \cdot R_{z}(\theta) \cdot R_{y}(\beta) \cdot R_{x}(\alpha) \cdot T
$$

In this formula, $R(\theta)$ is the required transformation matrix. The results are calculated by the following calculations: 


$$
\begin{aligned}
& T=\left[\begin{array}{cccc}
1 & 0 & 0 & -x_{1} \\
0 & 1 & 0 & -y_{1} \\
0 & 0 & 1 & -z_{1} \\
0 & 0 & 0 & 1
\end{array}\right], \quad R_{x}(\alpha)=\left[\begin{array}{cccc}
1 & 0 & 0 & 0 \\
0 & \frac{c}{d} & -\frac{b}{d} & 0 \\
0 & \frac{b}{d} & \frac{c}{d} & 0 \\
0 & 0 & 0 & 1
\end{array}\right], \\
& \begin{array}{c}
d=\sqrt{b^{2}+c^{2}}, R_{x}^{-1}(\alpha)=R_{x}(-\alpha), R_{y}^{-1}(\beta) \\
R_{y}(\beta)=\left[\begin{array}{cccc}
d & 0 & -a & 0 \\
0 & 1 & 0 & 0 \\
a & 0 & d & 0 \\
0 & 0 & 0 & 1
\end{array}\right], R_{z}(\theta)=\left[\begin{array}{cccc}
\cos \theta & -\sin \theta & 0 & 0 \\
\sin \theta & \cos \theta & 0 & 0 \\
0 & 0 & 1 & 0 \\
0 & 0 & 0 & 1
\end{array}\right],
\end{array}
\end{aligned}
$$

Using the formula (2), the result $R(\theta)$ was obtained. It will display correct result transforming the model using $R(\theta)$.

\subsubsection{Textures}

Mapping errors occurred during the development process. Developers need make sure the texture files use the unix line endings not windows-style line endings so that ARToolKit can read texture files correctly. If the texture is chaotic, it may be caused by incorrectly reading the y-coordinate of the texture picture. The solution is to flip the map vertically or modify the program to swap the y values.

\subsection{Technical details of color image marker scheme}

In order to enhance user experience, this article also uses color pictures of cultural relics as a marker for the development.

\subsubsection{Markers creation}

Developers can use jpeg files of color images and the program "genTexData" provided by ARToolKit to generate natural feature tracking (NFT) data files for recognising, matching and tracking markers.

\subsubsection{Addition of 3D models}

This article will use the ARToolKit example project "nftBookProj" to describe and write a new custom application program.

In this example, the OpenSceneGraph development package is used to load virtual 3D model files. If you need add support for obj format files in your program, you need to do the following:

- Add the code line in "osgPlugin.h" file: USE_OSGPLUGIN (obj)

- Modify "Android.mk" file and add code "osgdb_obj" to the end of lines beginning with "LOCAL_STATIC_LIBRARIES + =" and "ARTOOLKIT_LIBS + ="

In this example project, a text file named "marker.dat" and a text file named "objects.dat" in the "assets/Data" folder are used to set the markers information and the virtual 3D model files information, respectively.

\subsubsection{Models transform}

The developer can takes $R(\theta)$ obtained in section 3.1.3 or other transformation matrix into the function "arOSGSetModelLocalPose" in the native cpp file to transform a model.

\section{Experimental results}

Table 1: Information of models and data files.

\begin{tabular}{ccccccc}
\hline $\begin{array}{c}\text { model } \\
\text { name }\end{array}$ & $\begin{array}{c}\text { vertice } \\
\text { number }\end{array}$ & $\begin{array}{c}\text { face } \\
\text { number }\end{array}$ & $\begin{array}{c}\text {.obj file } \\
\text { size }\end{array}$ & $\begin{array}{c}\text { texture file } \\
\text { size }\end{array}$ & $\begin{array}{c}\text { black and white } \\
\text { marker data files size }\end{array}$ & $\begin{array}{c}\text { color image marker } \\
\text { data files size }\end{array}$ \\
\hline FO & 10730 & 20000 & $1.76 \mathrm{MB}$ & $378 \mathrm{~KB}$ & $12.1 \mathrm{~KB}$ & $155 \mathrm{~KB}$ \\
HEZI & 10002 & 20000 & $1.66 \mathrm{MB}$ & $384 \mathrm{~KB}$ & $12.1 \mathrm{~KB}$ & $57.7 \mathrm{~KB}$ \\
YC & 10000 & 20000 & $1.68 \mathrm{MB}$ & $149 \mathrm{~KB}$ & $12.1 \mathrm{~KB}$ & $163 \mathrm{~KB}$ \\
YU & 10033 & 20000 & $1.67 \mathrm{MB}$ & $131 \mathrm{~KB}$ & $12.1 \mathrm{~KB}$ & $206 \mathrm{~KB}$ \\
ZHUAN & 10002 & 20000 & $1.66 \mathrm{MB}$ & $218 \mathrm{~KB}$ & $12.3 \mathrm{~KB}$ & $258 \mathrm{~KB}$ \\
\hline
\end{tabular}


The system is developed based on ARToolkit v5.3.2. This experiment uses a Smartisan T1 phone to collect data and to view the display. The following is the mobile phone parameters: CPU Qualcomm Snapdragon 801, Cores Quad-core 2.5 GHz, GPU Adreno 330, RAM 2G, Rear camera 12.78 million pixels, the screen resolution of 1920x1080 pixels, Operating System Android 4.4.2.

In this experiment, five models were used for test. Table 1 shows the relevant information about each model and corresponding data files.

\subsection{Black and white marker scheme}

The display of App using the black and white marker scheme is shown in Fig. 3 to 6.

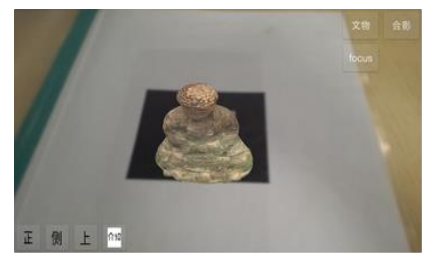

Fig. 3: Identify markers.

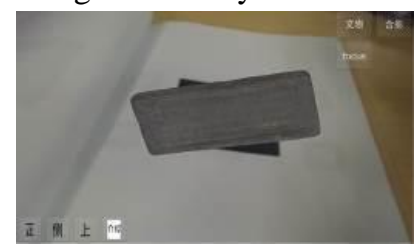

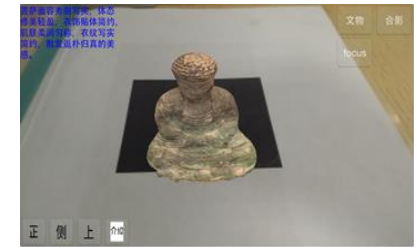

Fig. 4: Text and voice introduction.

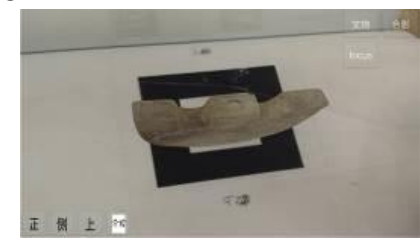

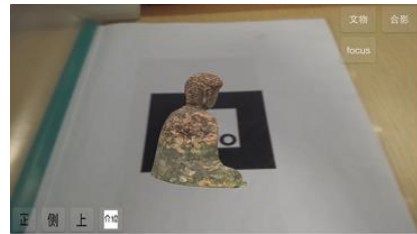

Fig. 5: Rotate the 3D model.

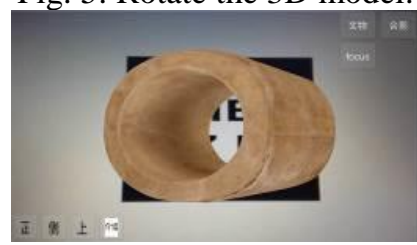

Fig. 6: Display different 3D models.

\subsection{Color image marker scheme}

The display of App using the color image marker scheme is shown in Figs. 7 to 10

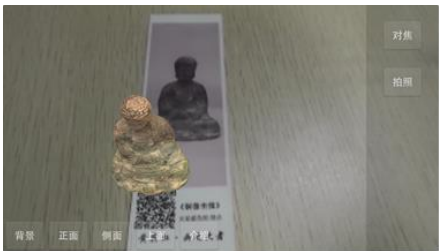

Fig. 7: Identify markers.

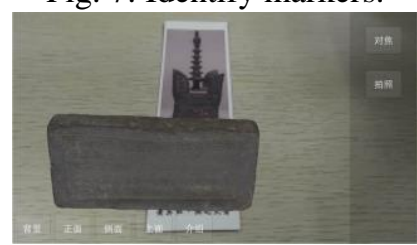

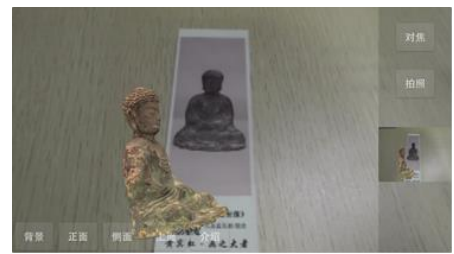

Fig. 8: Photo preview.

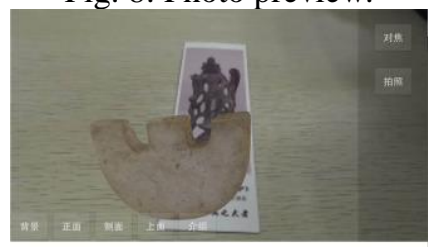

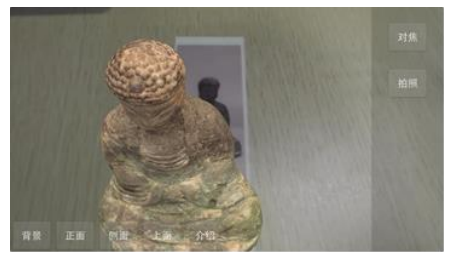

Fig. 9: Scale the 3D model.

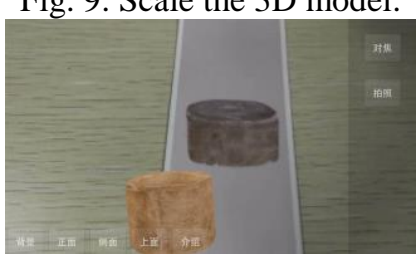

Fig. 10: Display different 3D models.

\subsection{Time of loading}

Because the size of data files is different about two schemes, two Apps' startup time for reading and loading data files is different. Make a comparative experiment by changing the number of markers and models in the App. The data obtained are shown in Table 2.

Table 2: Two scheme's load time.

\begin{tabular}{ccc}
\hline model number & $\begin{array}{c}\text { black and white marker scheme load } \\
\text { time(s) }\end{array}$ & $\begin{array}{c}\text { color image marker scheme load } \\
\text { time(s) }\end{array}$ \\
\hline 5 & 1.216 & 4.63 \\
10 & 2.464 & 9.42 \\
15 & 3.702 & 12.66 \\
20 & 4.874 & 21.74 \\
\hline
\end{tabular}

As can be seen from table above, Loading the same number of models, App using black and white marker scheme used significantly less time than App using color image marker scheme. 


\subsection{System application}

The system was used in conjunction with the WeChat self-help bookmark printing terminal provided by Zhejiang provincial museum. The use flow chart of this terminal is shown in Fig. 11. Four Steps for use as follows: Step 1, connect your phone to the Zhejiang provincial museum free Wi-Fi; Step 2, follow the official account of Zhejiang provincial museum in WeChat; Step 3, click on the printing bookmarks link in the official account; Step 4, select your favorite picture and print it.

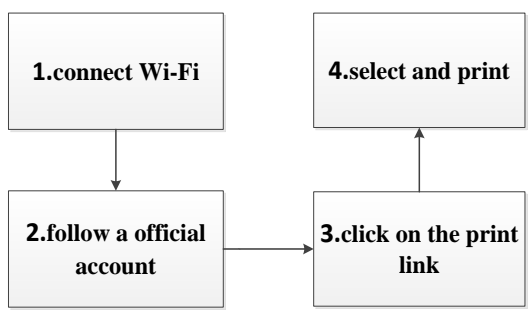

Fig. 11: Use flow chart of WeChat self-help bookmark printing terminal.

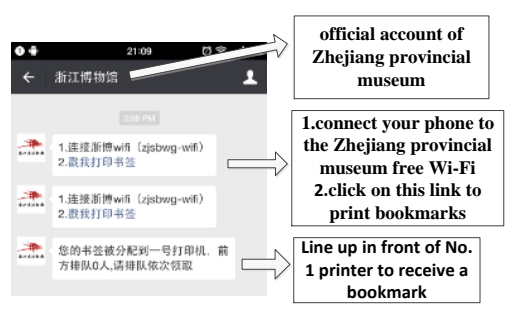

Fig. 12: WeChat interface of the selfhelp printing system.

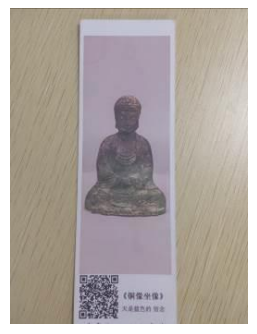

Fig. 13: Bookmark.

Fig. 12 shows the WeChat interface of self-help printing system. Fig. 13 shows a bookmark printed by this terminal.

\section{Conclusions}

The attractiveness of the museum mainly comes from two aspects. One is the resources of museum exhibits, the second is the way and means to display exhibits [6]. Resources are relatively stable, but the way can be constantly updated.

In this paper, we propose a kind of virtual exhibition system developed by using hand-held AR technology to solve the contradiction between taking pictures and order maintain in museums.

In order to shorten the system startup load time, we plan to use multi-threaded way to improve the system. The way is that system main process starts the application interface and works, while another process in the background continues to load the model. For the current selfie needs in museums, we will further research and development a selfie system combined virtual and real scenes. In the following system, visitors can take pictures with exhibits and virtual scenes. That will enhance visitors experience in museums.

\section{Acknowledgements}

Thanks for the support of the Zhejiang Natural Science Foundation project(LY13F020050) and Zhejiang cultural relics protection technology project "research and development on virtual and real image dynamic synthesis technology in mobile phone and this technology's innovative application in the museum" for this project.

\section{References}

[1] Bo Ren, Tao Guan, Lijun Li, et al. Exploiting and Applying of Augmented Reality System Based on ARToolKit[J]. Computer Systems Applications, 2006, 29(1):81-84.

[2] Ke Qiu. The Augmented Reality Used in the Museums. Digital Museum. Beijing: Publishing House of Electronics Industry, 2015: 12-19.

[3] Kato H. Inside ARToolKit. http://campar.in.tum.de/twiki/pub/Far/AugmentedRealityPraktikumSoSe2004/ART02Tutorial.pdf

[4] Kato H, Billinghurst M. Marker tracking and HMD calibration for a video-based augmented reality conferencing system. international symposium on mixed and augmented reality, 1999: 85-94

[5] Donald Hearn, M. Pauline Baker, Warren R. Carithers. Computer Graphics with OpenGL Fourth Edition. Beijing: Publishing House of Electronics Industry, 2014.

[6] Xiongfei Chen. The attraction of the museum. Chinese Museum, 1997(1):10-15. 